ARTICLE

\title{
Dysferlin-mediated phosphatidylserine sorting engages macrophages in sarcolemma repair
}

\author{
Volker Middel ${ }^{1}$, Lu Zhou ${ }^{2,3}$, Masanari Takamiya1, Tanja Beil', Maryam Shahid ${ }^{1}$, Urmas Roostalu4 \\ Clemens Grabher ${ }^{1}$, Sepand Rastegar ${ }^{1}$, Markus Reischl ${ }^{5}$, Gerd Ulrich Nienhaus ${ }^{1,2,3,6}$ \& Uwe Strähle ${ }^{1}$
}

Failure to repair the sarcolemma leads to muscle cell death, depletion of stem cells and myopathy. Hence, membrane lesions are instantly sealed by a repair patch consisting of lipids and proteins. It has remained elusive how this patch is removed to restore cell membrane integrity. Here we examine sarcolemmal repair in live zebrafish embryos by real-time imaging. Macrophages remove the patch. Phosphatidylserine (PS), an 'eat-me' signal for macrophages, is rapidly sorted from adjacent sarcolemma to the repair patch in a Dysferlin (Dysf) dependent process in zebrafish and human cells. A previously unrecognized arginine-rich motif in Dysf is crucial for PS accumulation. It carries mutations in patients presenting with limb-girdle muscular dystrophy 2B. This underscores the relevance of this sequence and uncovers a novel pathophysiological mechanism underlying this class of myopathies. Our data show that membrane repair is a multi-tiered process involving immediate, cell-intrinsic mechanisms as well as myofiber/macrophage interactions.

\footnotetext{
${ }^{1}$ Institute of Toxicology and Genetics, Karlsruhe Institute of Technology (KIT), PO Box 3640, 76021 Karlsruhe, Germany. ${ }^{2}$ Institute of Applied Physics, Karlsruhe Institute of Technology (KIT), Wolfgang-Gaede-Straße 1, 76131 Karlsruhe, Germany. ${ }^{3}$ Institute of Nanotechnology, Karlsruhe Institute of Technology (KIT), PO Box 3640, 76021 Karlsruhe, Germany. ${ }^{4}$ Institute of Inflammation and Repair, The University of Manchester, Oxford Road, Manchester M13 9PL, UK.

${ }^{5}$ Institute for Applied Computer Science, Karlsruhe Institute of Technology (KIT), PO Box 3640, 76021 Karlsruhe, Germany. ${ }^{6}$ Department of Physics, University of Illinois at Urbana-Champaign, 1110 West Green Street, 61801 Urbana, Illinois, US. Correspondence and requests for materials should be addressed to U.S. (email: uwe.straehle@kit.edu).
} 
S keletal muscle cells are prone to plasma membrane lesions under physiological levels of mechanical stress. To prevent cell death and to avoid muscle regeneration with concomitant depletion of stem cell pools, lesions are rapidly sealed by a repair patch consisting of proteins and lipids ${ }^{1,2}$. The transmembrane protein Dysf plays a key role in restoring cell integrity. Humans with mutations in the DYSF gene acquire limb-girdle muscular dystrophy type 2B (LGMD2B), Miyoshi myopathy or distal myopathy with anterior tibialis onset ${ }^{3,4}$. Dysf binds phosphatidylserine (PS) in a $\mathrm{Ca}^{2+}$-dependent manner through its $\mathrm{C} 2$-domains ${ }^{5}$. Mini-Dysf generated by $\mathrm{Ca}^{2+}$. dependent calpain proteases and comprising the last two C2domains of the full length mammalian protein appears to be the isoform of Dysf active in membrane repair ${ }^{6}$. In zebrafish, deletion of the C2 domains generated an isoform that is still able to accumulate at the site of lesion ${ }^{7}$. How Dysf precisely acts in membrane repair has remained elusive. Other players in membrane repair are annexins (AnxAs), which associate into multimeric complexes in a $\mathrm{Ca}^{2+}$-dependent manner at the lesion $^{8-10}$. These complexes are built by homo and heteromeric interactions of AnxAs and include also Dysf and possibly other repair proteins and lipids ${ }^{7-10}$. The distinct temporal order of proteins' arrival in the repair patch suggests that a specific structure of the protein-lipid matrix is required for its function ${ }^{7}$. Knock-down of Dysf or AnxA6 translation caused a malformed repair patch and double morphants even showed leakage of cytoplasmic components into the extracellular environment ${ }^{\text {. The }}$. The origin of the membrane material forming the repair patch is unclear. Lysosomes as well as endosomes were suggested to contribute to membrane repair in mammalian cell systems ${ }^{11,12}$. However, none of the tested intracellular vesicles marked by Laptm4a, Lamp1, Lamp2, Rab1a, Rab5a, Rab6a, Rab7 and Rab27a contribute significantly to repair patch formation in zebrafish myofibers ${ }^{7}$. Although mechanisms like membrane shedding, exocytosis and endocytosis were discussed, a still unresolved issue is how the repair patch is removed to restore the plasma membrane ${ }^{2}$.

We have employed live fluorescence imaging including single-molecule based techniques to study membrane repair in muscle cells in real time in zebrafish embryos and human cells. We provide evidence that macrophages remove the repair patch from damaged cells involving selective enrichment of PS at the lesion. Strikingly, Dysf mediates PS transport from adjacent sarcolemma to the repair patch through a five amino acid (AA)-motif close to its transmembrane (TM) domain, harbouring a point mutation in certain LGMD2B patients.

\section{Results}

Macrophages remove repair patch. To assess up to which size a sarcolemmal lesion can still be repaired, we damaged the sarcolemma of single myofibers in the somitic musculature coexpressing plasmids encoding $\mathrm{Ca}^{2+}$ sensing GCaMP5A (ref. 13) together with mOrange-tagged AnnexinA2a (AnxA2a-mO) ${ }^{7}$. After a transient increase of $\mathrm{Ca}^{2+}$ ions at the lesion, a patch rapidly formed. All fibres with lesions $\leq 4 \mu \mathrm{m}$ survived membrane damage whereas lesions $\geq 4 \mu \mathrm{m}$ were associated with cell death (Supplementary Fig. 1a-g, Supplementary Movie 1, 2).

Frequently, we observed a motile cell appearing at the repair patch. We hypothesized neutrophils or macrophages were attracted to these sites and utilized $\operatorname{Tg}($ mpeg1:GFP) and $\operatorname{Tg}(l y s C: d s R e d)$ marking macrophages ${ }^{14}$ and neutrophils ${ }^{15}$, respectively. The membranes of AnxA2a-mO expressing myofibers of transgenic fish were injured and leucocyte migration was monitored. Macrophages were recruited to injured myofibers (Fig. 1a-c, Supplementary Movie 3) in 50\% of the cases within $16.4 \pm 11.6 \mathrm{~min}(n=92)$ after injury. In long-term $(16-20 \mathrm{~h})$ time-lapse experiments, $92 \%$ of the damaged myofibers had been visited by macrophages $(n=143)$ (Supplementary Fig. 2a-c,g; Supplementary Movie 4). While myofibers with small lesions $(\leq 4 \mu \mathrm{m})$ attracted on average $1.29 \pm 0.2$ macrophages $(n=53)$, about twice as many macrophages $(2.5 \pm 1.4, n=87)$ were encountered at cells with lesions $\geq 4 \mu \mathrm{m}$. Macrophages were observed to ingest parts of the repair patch in surviving cells with lesion smaller than $4 \mu \mathrm{m}$ (Fig. 1a,c). In contrast, macrophages phagocytosed entire myofibers with lesions $>4 \mu \mathrm{m}$ (Supplementary Fig. 2a-c). Neutrophils were detected only infrequently $(7 \%, n=126)$ at laser induced membrane lesions and did not participate in phagocytosis of dead myofibers. In contrast, sterility-compromised stabwounds inflicted by insertion of a glass needle into the somitic musculature attracted neutrophils in all cases $(n=23)$ similar to tailfin cuts $(n=12$; Supplementary Fig. 2h).

To enquire if macrophages are crucial for repair patch removal, we employed an established morpholino knock-down strategy ${ }^{16-18}$. By triple injection of morpholinos directed against gcsfr, pul and irfo mRNAs, we eliminated $T g(m p e g 1: G F P)$ expressing macrophages. In controls (Fig. 1d, $n=37$ ), the repair patch was removed in $62 \%$ of the damaged cells, whereas, in all morphants (Fig. 1d, $n=25, P<10^{-8}$ ), the patch persisted beyond the observation time $(20 \mathrm{~h})$. Myofibers damaged beyond repair were not phagocytosed in the morphants (Supplementary Fig. $2 \mathrm{~d}-\mathrm{g}$, $n=20$ ), whereas $92 \%$ were removed within $20 \mathrm{~h}$ in controls (Supplementary Fig. 2a-c, g, $n=12 ; P<10^{-8}$ ). We obtained the same results when we analysed a Crispr/Cas9 generated loss-of-function mutation in irf8 (Fig. 1e) underscoring that the observed morpholino effects were specific. Taken together, these findings strongly suggest a crucial and novel role of macrophages for selectively removing the repair patch at membrane lesions.

Fast and selective PS accumulation. Exposure of PS to the extracellular space can trigger phagocytosis by macrophages ${ }^{19}$. To investigate, whether PS accumulates in the repair patch, we co-expressed the stereo-specific and $\mathrm{Ca}^{2+}$-independent PS sensor LactAdherinC2:GFP (LactC2:GFP) ${ }^{20}$ together with AnxA2a-mO. The PS sensor accumulated earlier than AnxA2a-mO (Fig. 2a, Supplementary Fig. 3a; Supplementary Movie 5). Dysf fused to mOrange (Dysf-mO ${ }^{7}$ accumulated at the same pace as LactC2:GFP (Fig. 2b, Supplementary Fig. 3b) or TopFluor-PS (ref. 21) at membrane lesions (Supplementary Fig. 3c). Clearly, PS is one key component of the early repair patch.

We next analysed the selectivity of PS accumulation. Membrane tethered CAAX-mCherry ${ }^{22}$ accumulated more slowly (Fig. 2c, Supplementary Fig. 3d) compared with LactC2:GFP (Fig. 2c). Lyn-tailed mCherry ${ }^{23}$, another membrane marker, showed no accumulation at the lesion (Fig. 2d, Supplementary Fig. 3e). We also tested lipid sensors recognizing phosphatidylinositol 4,5-bisphosphate $(\mathrm{PI}(4,5) \mathrm{P} 2$, GFP- $\times$ PH(PLC $))^{24}$, phosphatidylinositol (3,4,5)-trisphosphate (PIP3; pEGFP::2FYVE-GFP) ${ }^{25}$, phosphatidylinositol 3,4-bisphosphate (PI(3,4)P2, AKT-PH: EGFP) ${ }^{25,26}$ and a fluorescently tagged cholesterol (BODIPYcholesterol) ${ }^{27}$. Except for cholesterol (Fig. 2h, Supplementary Fig. 3i), none of the lipid markers accumulated at levels comparable to PS (Fig. 2e-g, Supplementary Fig. 3f-h).

To assess whether PS is presented to macrophages at the repair patch, we expressed the secreted PS sensor secA5-YFP (ref. 28) and injured non-expressing myofibers (Fig. 2i-l). Hence, secA5-YFP was exclusively supplied to the lesion patch from the extracellular space (Fig. 2j,k). secA5-YFP was enriched at the lesion (Fig. 2l, Supplementary Movie 6) demonstrating that PS is present at the extracellular surface of the lesion patch. 

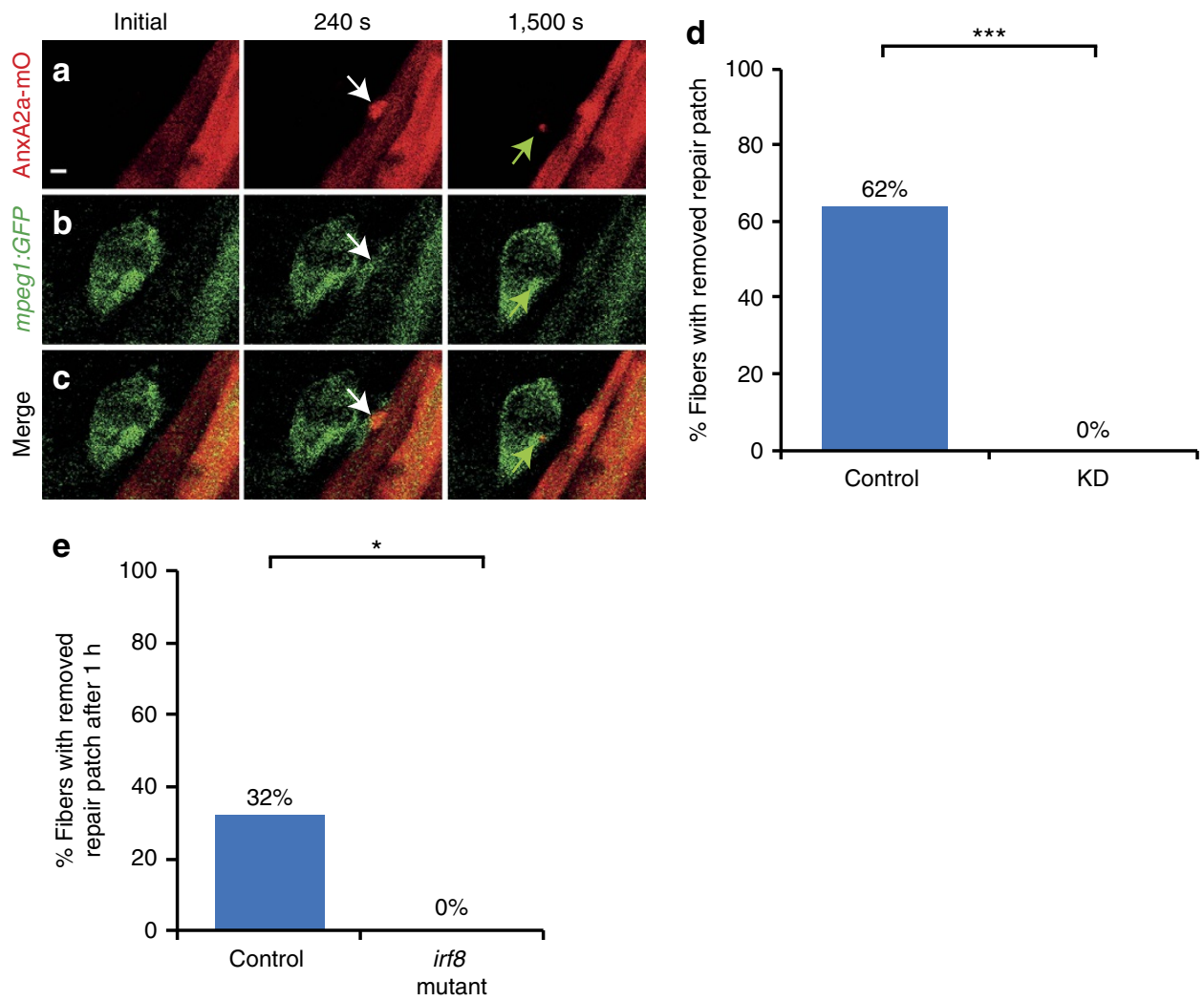

Figure 1 | Macrophages remove repair patch. (a-c) Repair patch (AnxA2a-mO, a, white arrow) and macrophages (mpeg1:GFP, b, white arrows) before (Initial), $240 \mathrm{~s}$ and 1,500 s after wounding. A macrophage ingests parts of repair patch (green arrows). (c) Merged views of a,b. Note the bleed-through of AnxA2a-mO into the GFP channel (b). (d) Repair patch removal in control (left, $n=37$ ) and macrophage-depleted embryos (KD, right, $n=25$, Fisher's exact test $P<10^{-8}$ ) over $20 \mathrm{~h}$. (e) Crispr/Cas9 knock-out of irf8 impairs repair patch removal. In controls, the repair patch was removed in $32 \%$ of injured myofibers $(n=19)$ within one hour after injury. In the irf8-KO embryos the patch was present in all cases of injured myofibers examined ( $n=15)$. This suggests that morpholino triple knock-down is inefficient and the same effect on macrophages can be achieved by elimination of irf8 at the stage analysed. Significance was checked with Fishers exact test at $P<0.05(P=0.023895)$. Scale bar, $4 \mu \mathrm{m}$.

We reasoned that secA5-YFP masks PS at the lesion patch and thereby delays phagocytosis. Indeed, phagocytosis was delayed in embryos expressing secA5-YFP $(n=19 ; 349 \pm 241 \mathrm{~min})$ in comparison with Dysf-mO $(n=23 ; 108 \pm 104 \mathrm{~min} ; P<0.00001)$ or AnxA2a-mO $(n=22 ; 124 \pm 79 \mathrm{~min} ; P<0.00001)$ expressing fibres (Fig. 2m). Taken together, PS rapidly and selectively accumulates in the lesion patch, is presented to the outside, and masking of PS delays phagocytosis. All these observations clearly support the notion that PS marks the repair patch for phagocytosis by macrophages.

Dysf is required for PS accumulation. Since Dysf fragments and LactC2:GFP accumulated at similar speeds, we tested whether Dysf plays an active role in PS accumulation. We inflicted membrane wounds in myofibers expressing LactC2:GFP in combination with morpholino (MO) knock-down of endogenous Dysf ${ }^{7}$. Injecting a $5 \mathrm{bp}$ mismatch contr-MO caused normal PS accumulation (Fig. 3a,c). Injection of dysf-MO, however, abolished PS recruitment (Fig. 3b,c, Supplementary Fig. 4a). Co-injection of $d y s f-M O$ together with mOrange1-DysfC ${ }^{7}$ restored accumulation of PS (Fig. 3c, Supplementary Fig. 4a). AnxA6 is required for the formation of a tight lesion patch ${ }^{7}$. In anxa6 morphants, PS accumulation was indistinguishable from that of controls (Fig. 3d, Supplementary Fig. 4b). Furthermore, $d y s f$-mutant embryos, in contrast to wild-type siblings showed significantly reduced PS accumulation (Supplementary Fig. 4c-f).
Since a 74 AA C-terminal fragment of Dysf ${ }^{7}$ was sufficient to restore PS translocation towards the lesion, we next asked which region in Dysf (Fig. 3e) mediates PS accumulation. Thus, we shortened the fragment further to a predicted amphipathic helix ${ }^{29}$, residing N-terminally of and within the putative TM domain (Fig. 3e, zfWRRFK-TM-C). zfWRRFK-TM-C localized to the Z-lines and sarcolemma and translocated to the repair patch after membrane injury (Fig. 3f,h). Deletion of 14 AAs from the C-terminus (zfWRRFK-TM) did not affect accumulation (Fig. 3e,h, Supplementary Fig. 4g). However, removal of 5 AAs $\mathrm{N}$-terminally led to mislocalization in intact cells and failure to accumulate (Fig. 3g,h, Supplementary Fig. 4g). Strikingly, PS accumulation was not rescued in $d y s f$-morphants co-injected with $z f T M-C$, while $z f W R R F K-T M-C$ rescued PS enrichment (Fig. 3i, Supplementary Fig. 4h), similar to $d y s f$-mutant embryos (Supplementary Fig. 4i,j). In conclusion, this 5-AA motif which is not related to the reported PS binding C2-domains of Dysf (ref. 5) is essential for the selective accumulation of PS at the repair patch.

Dysf mediates PS relocation. To assess the dynamics of Dysf-mO, we employed fluorescence loss in photobleaching (FLIP). Dysf-mO expressing myofibers were imaged every second for $118 \mathrm{~s}$ under a spinning disk laser scanning microscope (Fig. 4a) and damaged by 405-nm laser irradiation. The light exposure also caused substantial bleaching at the site of lesion (Fig. 4a). As a control, we irradiated with $561 \mathrm{~nm}$ instead of $405 \mathrm{~nm}$ laser light, which only bleaches but does not damage the 

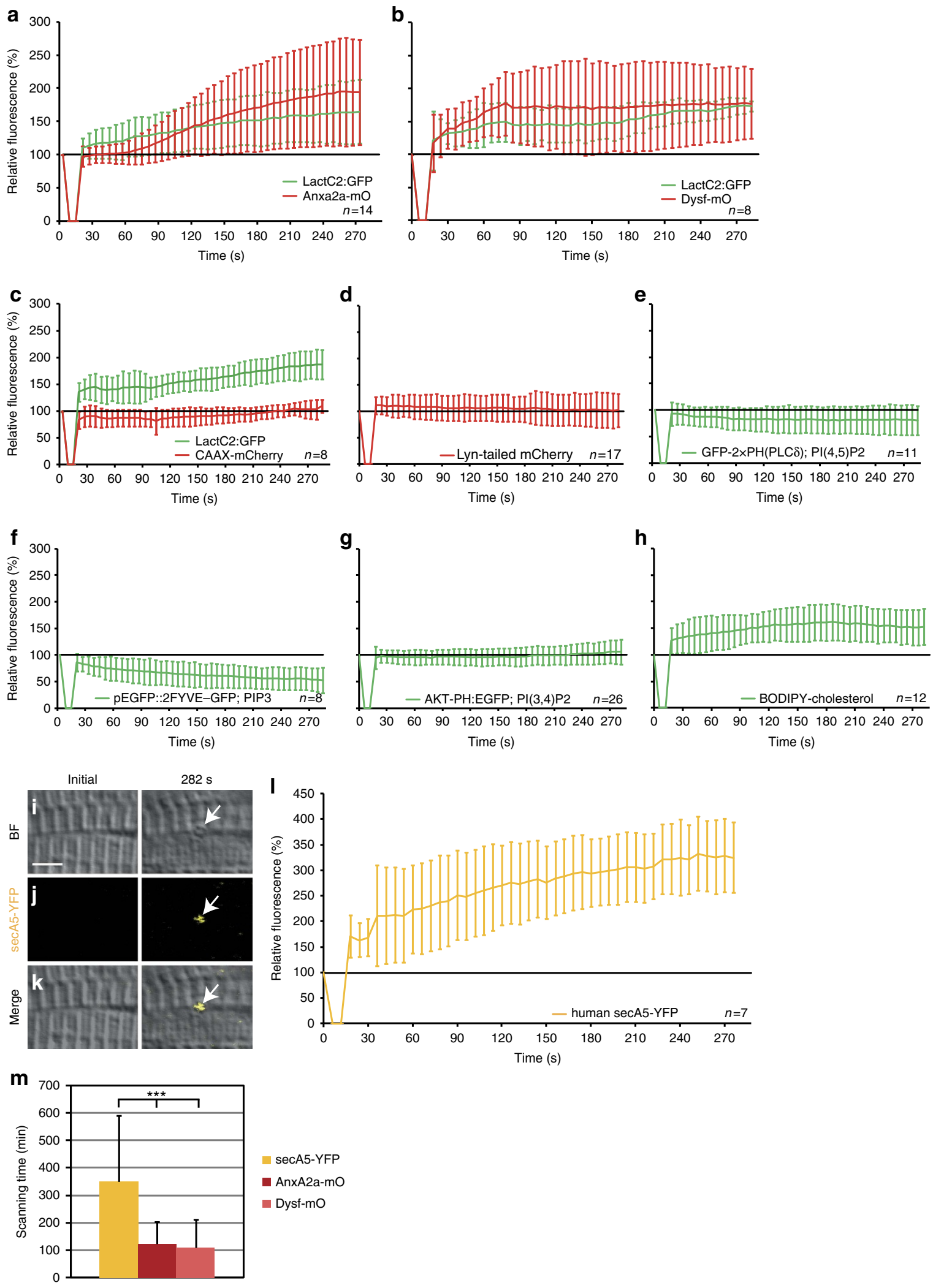

Figure 2 | Phosphatidylserine (PS) is sorted to the repair patch. (a-h) Real-time analysis of enrichment of PS (LactC2:GFP (a-c)), PI(4,5)P2 (GFP$2 \times \mathrm{PH}(\mathrm{PLC} \delta)(\mathbf{e})$ ), PIP3 (pEGFP::2FYVE-GFP (f)), PI(3,4)P2 (AKT-PH:EGFP (g)) and cholesterol (BODIPY-cholesterol (h)), relative to AnxA2a-mO (a), Dysf-mO (b) and membrane markers CAAX-mCherry (c), Lyn-tailed mCherry (d). The reporter fluorescence is expressed as percentage (mean \pm s.d.) relative to the level before injury. (i-m) PS is presented on the extracellular side of the repair patch. (i-I) Extracellularly supplied secA5-YFP was enriched at membrane on lesioning (i-k, arrows). secA5-YFP expressing myofibers are outside of the field of view. (I) Kinetics of secA5-YFP at the repair patch. (m) Macrophage scanning-time ( $+/$ - s.d.) of myofibers expressing secreted secA5-YFP (yellow, $n=19$ ), AnxA2a-mO (dark red, $n=22$ ) or Dysf-mO (light red, $n=23$; Student $t$-test $P<0.001$ ) Scale bar, $4 \mu \mathrm{m}$. 

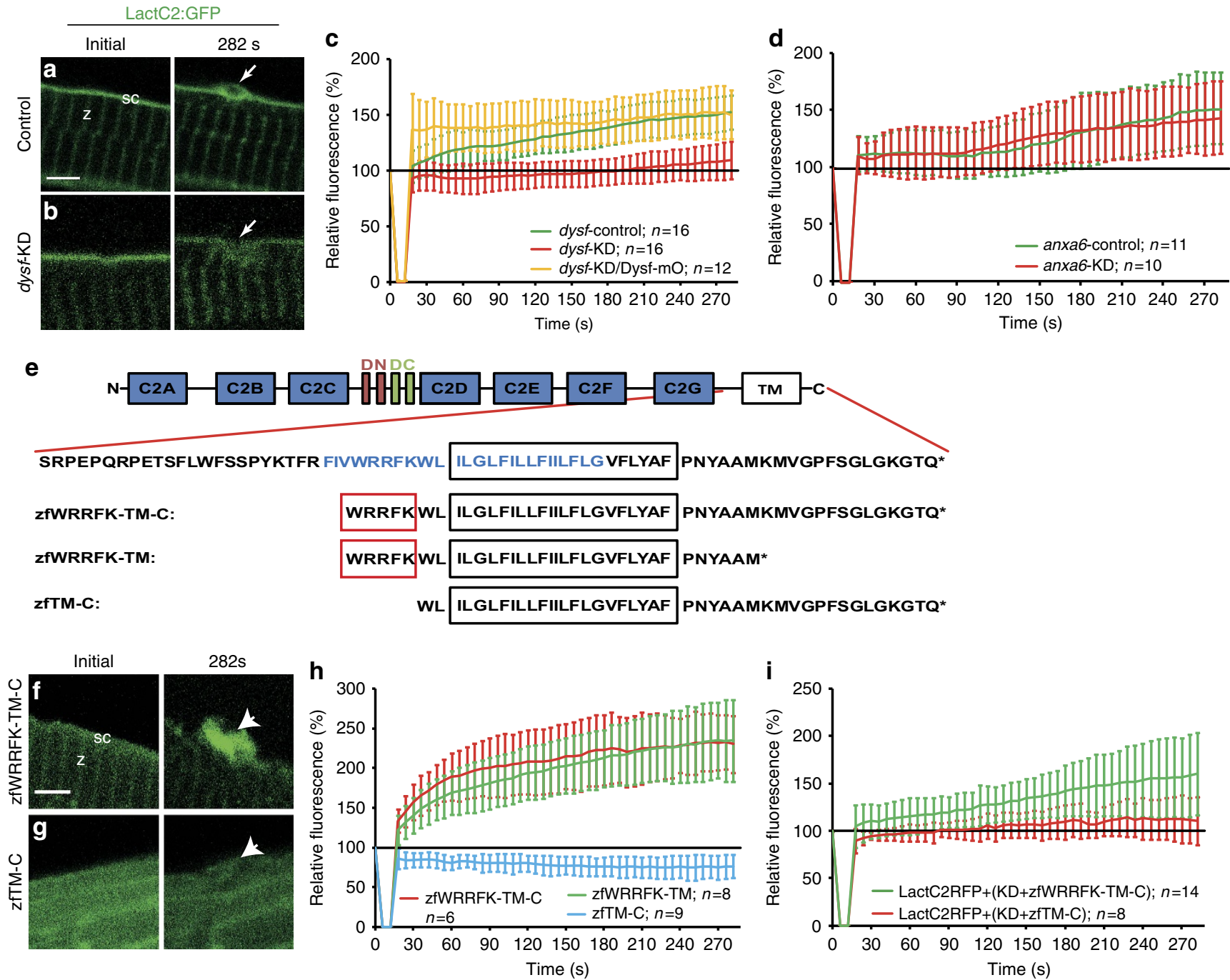

Figure 3 | Five amino acid motif in Dysf is required for PS accumulation. (a,b) Before wounding, LactC2:GFP (green) localized to the sarcolemma (sc; $\mathbf{a})$ and the Z-line ( $z ; a)$ both in control (a) and knock-down (dysf-KD; b) myofibers. On injury, LactC2:GFP accumulated in controls (a, arrow) but not in dysf-KD myofibers (b, arrow). (c) Kinetics of LactC2:GFP accumulation in control-KD (green), dysf-KD (red) and dysf-KD embryos co-injected with mOrange1-DysfC (Dysf-mO, yellow). Lack of PS accumulation in dysf-KD myofibers was rescued by Dysf-mO, translation of which is not inhibited by dysf morpholino. (d) Knock-down of anxa6 (anxa6-KD) had no effect on PS accumulation (red) compared with controls (anxa6-control, green). (e) Domain structure of Dysf. (blue AA: predicted amphipathic helix). (f,g), On damage zfWRRFK-TM-C accumulates in the repair patch ( $f$, arrow). No accumulation was observed for zfTM-C (g, arrow). (h) Accumulation kinetics of zfWRRFK-TM-C (red), zfWRRFK-TM (green) and zfTM-C (blue). A 5-AA motif (WRRFK, red box, e) is required for accumulation. (i) zfWRRFK-TM-C (red) but not zfTM-C (green) rescued PS accumulation in dysf-KD myofibers. In all charts, the change of fluorescence at the lesion is indicated as percentage relative to the undamaged state \pm s.d.). Scale bars, $4 \mu \mathrm{m}$ (a,g-h), $3 \mu \mathrm{m}$ (b).

membrane (Fig. 4b). After local bleaching using 561-nm irradiation, we observed a fluorescence intensity decrease in the region of interest adjacent to the bleached site followed by slow recovery (Fig. 4c). In contrast, after local bleaching and membrane damage by $405-\mathrm{nm}$ irradiation, the fluorescence intensity decreased faster and to a significantly lower level (Fig. 4c, Supplementary Fig. 5a). The faster kinetics and greater extent of fluorescence loss after membrane damage provide strong evidence that Dysf from adjacent, intact sarcolemmal regions is recruited to the site of lesion (Fig. 5a).

To directly visualize the movement of individual molecules to the wounded area, we performed super-resolution photoactivation localization microscopy, using green-to-red photoconversion of mEosFP (ref. 30). From these data, we analysed the displacements and speeds of $130 \pm 5$ single-molecule trajectories of mEosFP:zfWRRFK-TM-C along a line connecting the trajectory midpoint and the damage site in regions $2-15 \mu \mathrm{m}$ away from the wound (Fig. $4 \mathrm{~d}$ ). A parameter, $R_{\text {proj. }}$ was defined revealing random $\left(R_{\text {proj. }}=1\right)$ or net directional motion towards $\left(R_{\text {proj. }}>1\right)$ or away from $\left(R_{\text {proj. }}<1\right)$ the site of lesion (see supplementary experimental procedures). Figure $4 \mathrm{e}$ shows a super-resolved image of a mEosFP: zfWRRFK-TM-C labelled myofiber in zebrafish; the arrow indicates the site of damage. The resulting trajectories are shown in Fig. 4e. For zfWRRFK-TM-C, we observed a strong tendency to move towards the damage site $\left(R_{\text {proj. }}=1.92\right)$ and to accumulate at the membrane lesion (Fig. 4f). For the undamaged membrane region on the left (Fig. 4b), directional motion is absent $\left(R_{\text {proj }}=1.04\right)$, calculated with respect to a randomly chosen point (black dot). Control experiments with lipid-anchored CAAX-mEosFP did not display directional motion in the presence of damage (Fig. $4 \mathrm{f}, P<10^{-8}$ ), showing that directional motion of zfWRRFK-TM-C was not caused by overall membrane shifts towards the lesion. 

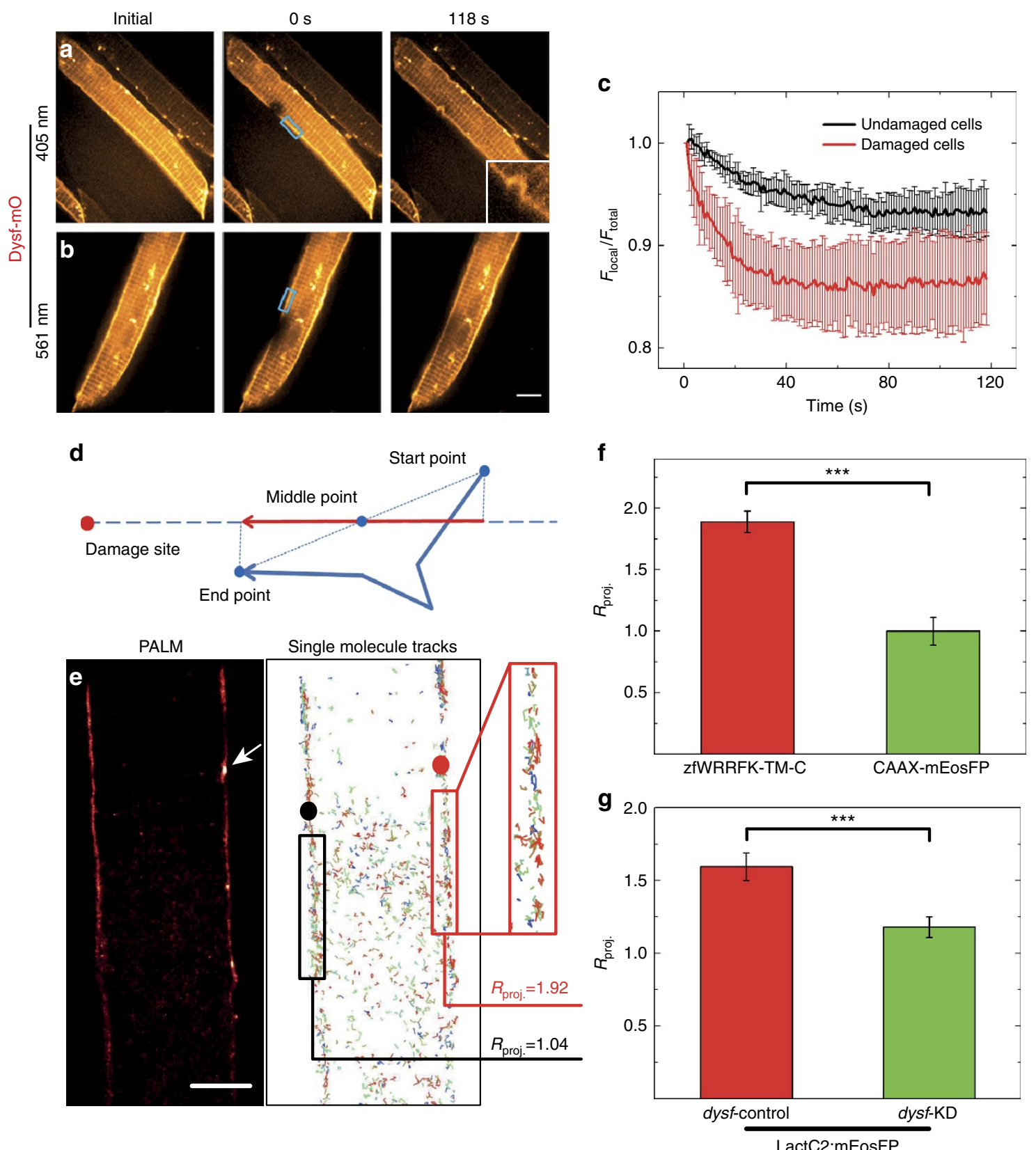

Figure 4 | Dysf facilitates PS translocation to site of lesion. (a-c) Fluorescence loss in photobleaching (FLIP) analysis on selected regions (blue boxes in a,b) of live Dysf-mO expressing myofibers. (a) Photobleaching and membrane damaging using $405 \mathrm{~nm}$ laser; (b) photobleaching only using $561 \mathrm{~nm}$ laser. (c) $561-\mathrm{nm}$ irradiation results in an intensity decrease by $6 \%$ (black curve); 405 -nm irradiation results in a more rapid decrease by $14 \%$ (red curve). (d-g) Single-molecule trajectory analysis of zfWRRFK-TM-C and controls after sarcolemmal damage. (d) Scheme showing the projection of a singlemolecule trajectory onto the line connecting the midpoint with the site of damage. (e) Super-resolution localization image of a mEosFP:zfWRRFK-TM-C labelled myofiber. The arrow indicates the site of lesion. Single-molecules trajectories calculated from the image data in e. Molecules near the lesion (red box) show a high tendency $\left(R_{\text {proj. }}=1.92\right)$ to move towards the lesion (red dot). In the undamaged sarcolemma (left), trajectories (black box) did not show directed motion towards the black dot $\left(R_{\text {proj. }}=1.04\right)$. (f) mEosFP:zfWRRFK-TM-C moved towards the lesion (red column); the control CAAX-mEosFP did not (green column; $P<10^{-8}$ ). ( $(\mathbf{g})$ The PS sensor LactC2:mEosFP moved towards the lesion in dysf-contr-KD embryos (red column) but not in dysf-KD embryos (green column; Student $t$-test $P<10^{-8}$ ). $n \geq 8$. Scale bar, $5 \mu \mathrm{m}$.

We performed additional single-molecule tracking experiments by using the PS sensor LactC2:mEosFP in dysf knock-down and control embryos. LactC2:mEosFP showed a much higher tendency to move towards the lesion in control than in dysf knock-down embryos (Fig. 4g, $P<10^{-8}$ ), further supporting our claim that Dysf plays a decisive role in translocating PS within the membrane to the damage site.
Dysf and PS accumulation require arginines. Double arginine (RR) motifs have previously been implicated in $\mathrm{Ca}^{2+}$-independent interaction with PS (ref. 31). Therefore, we tested the relevance of arginines within the WRRFK-motif (Fig. 3e) for accumulation of the Dysf and PS reporters. In contrast to zfWRRFK-TM-C, zfWAAFK-TM-C did not show any enrichment at the lesion (Fig. 5a, Supplementary Fig. 6a). Mutation of either one of the two arginines reduced translocation to the lesion (Fig. 5a, 

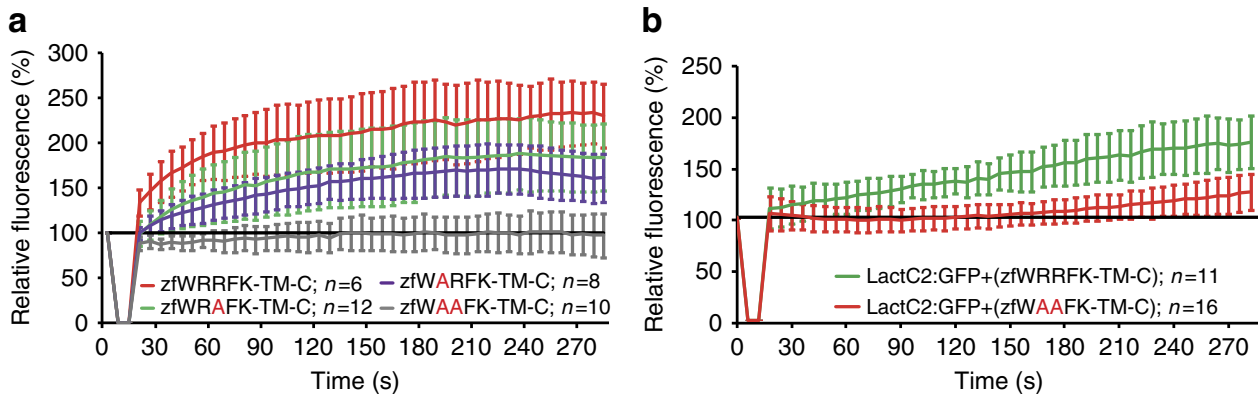

HeLa cells

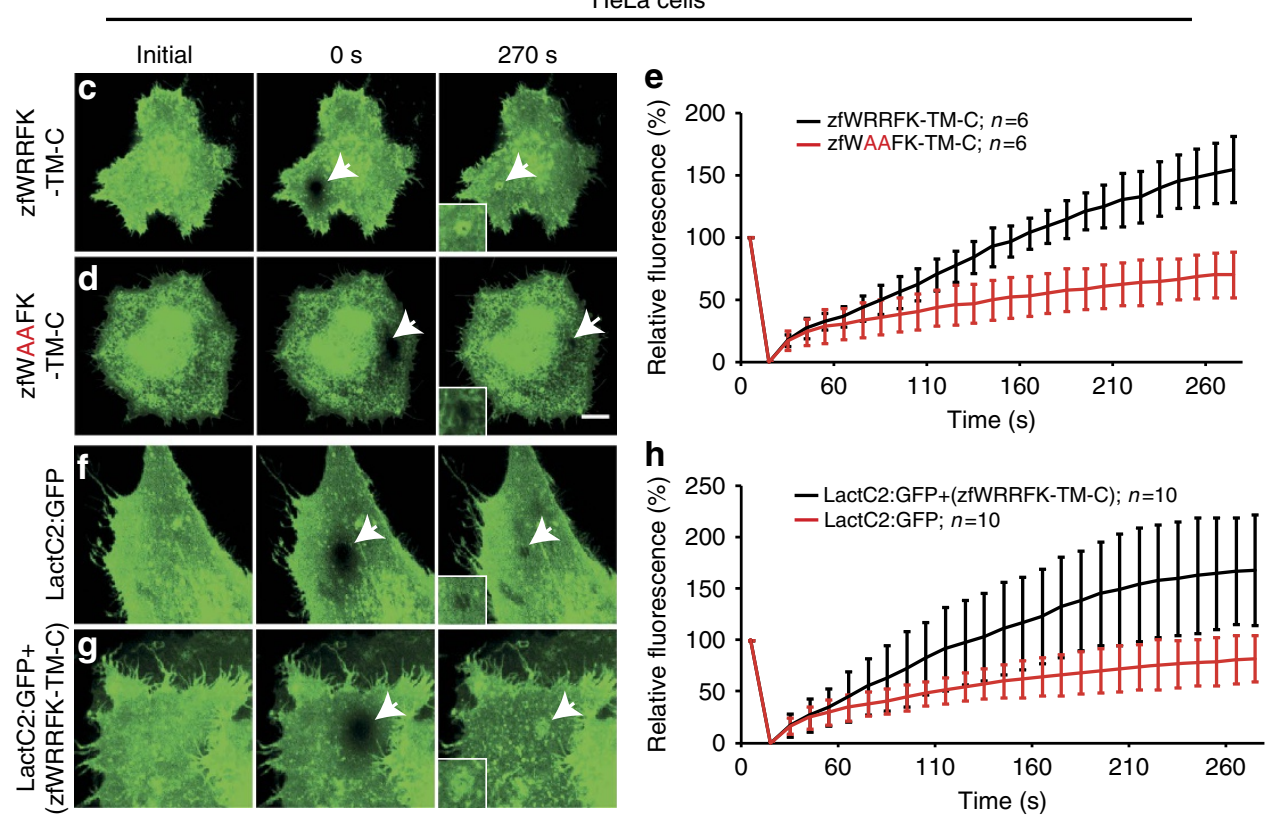

Figure 5 | The WRRFK-motif is required for accumulation of Dysf and PS. (a) After membrane damage, zfWRRFK-TM-C (red) but not zfWAAFK-TM-C (grey) accumulated rapidly at the lesion. The effect was reduced on exchange of the first (zfWARFK-TM-C; purple) or second arginine by alanine (zfWRAFK-TM-C; green). (b) PS (LactC2:GFP) enrichment in the repair patch of Dysf-KD myofibers co-expressing zfWRRFK-TM-C (green), but not of those co-expressing zfWAAFK-TM-C (red). (c-e) HeLa cells expressing zfWRRFK-TM-C were imaged before (c) and $0 \mathrm{~s}$ (c, arrow) and $270 \mathrm{~s}$ after membrane damage (c, arrows). zfWRRFK-TM-C markedly amassed at the lesion (c, arrow, inset; $\mathbf{e}$, black), whereas zfWAAFK-TM-C showed only baseline fluorescence recovery after photobleaching (d, arrows, inset; $\mathbf{e}$, red). (f-h) HeLa cells transfected with LactC2:GFP and imaged before (f) and 0 s (arrow) and $270 \mathrm{~s}$ after lesioning (arrow) showed no PS accumulation but only baseline recovery (f, arrow, inset; $\mathbf{h}$, red); in the presence of zfWRRFK-TM-C, LactC2:GFP accumulated within the repair patch ( $\mathbf{g}$, arrows, inset; h, black). The data in (a,b,e,h) are given as mean \pm s.e.m. $(n=>5)$ and scaled such that $100 \%$ corresponds to fluorescence from the same area before damaging. Scale bars, $10 \mu \mathrm{m}(\mathbf{c}, \mathbf{d})$ and $12.86 \mu \mathrm{m}(\mathbf{f}, \mathbf{g})$.

Supplementary Fig. 6a), demonstrating that both contribute to Dysf accumulation (Fig. 5a).

To enquire whether mutating the RR motif affects PS enrichment at the lesion, we knocked-down $d y s f$ and expressed LactC2:GFP together with either zfWRRFK-TM-C or zfWAAFK-TM-C. While zfWRRFK-TM-C rescued PS accumulation, $d y s f$-morphants expressing zfWAAFK-TM-C showed only partial rescue (Fig. 5b, Supplementary Fig. 6b).

PS accumulation in human cells. We tested whether zfWRRFK-TM-C accumulates in a heterologous system at injured cell membranes using human HeLa cells expressing very low levels of endogenous DYSF (ref. 32). Membrane damage in zfWRRFK-TM-C expressing HeLa cells (Fig. 5c) resulted in significant enrichment in the lesion patch (Fig. 5c,e), whereas zfWAAFK-TM-C showed no accumulation (Fig. 5d,e, Supplementary Fig. 6c). We examined whether the zebrafish Dysf fragment leads to enrichment of PS at the site of lesion in HeLa cells. Co-expression of LactC2:GFP and zfWRRFK-TM-C led to significant accumulation of PS at membrane lesions (Fig. 5f-h, Supplementary Fig. 6d-f). Undifferentiated C2C12 myoblasts showed LactC2:GFP accumulation only when coexpressing zfWRRFK-TM-C (Supplementary Fig. 6g-j). In contrast, when $\mathrm{C} 2 \mathrm{C} 12$ cells were differentiated to myotubes, which express high amounts of endogenous DYSF, LactC2: GFP showed enrichment at the lesion site in the absence of co-transfected zfWRRFK-TM-C (Supplementary Fig. 6k,l). Thus, the C-terminal fragment of zebrafish Dysf is sufficient to mediate PS accumulation at a membrane lesion in human cells, strongly suggesting that the underlying mechanisms are conserved between fish and humans.

Dysferlinopathy caused by mutation of RR motif. A sequence similar to the zebrafish motif WRRFK is present in human Dysf (Fig. 6a, WRRFR). Therefore, we tested whether human WRRFR-TM-C also would accumulate in zebrafish. Indeed, hWRRFR-TM-C showed high enrichment at the lesion (Fig. 6b,c). The double arginine mutant hWAAFR-TM-C showed 
a

ZfWRRFK-TM-C

hWRRFR-TM-C

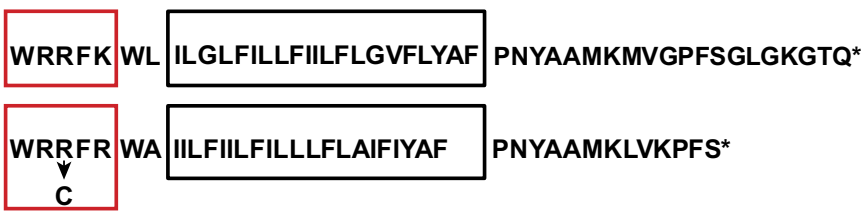

hWRRFR-TM-C

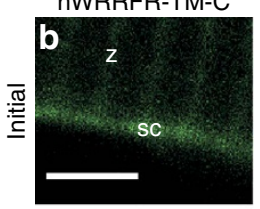

C
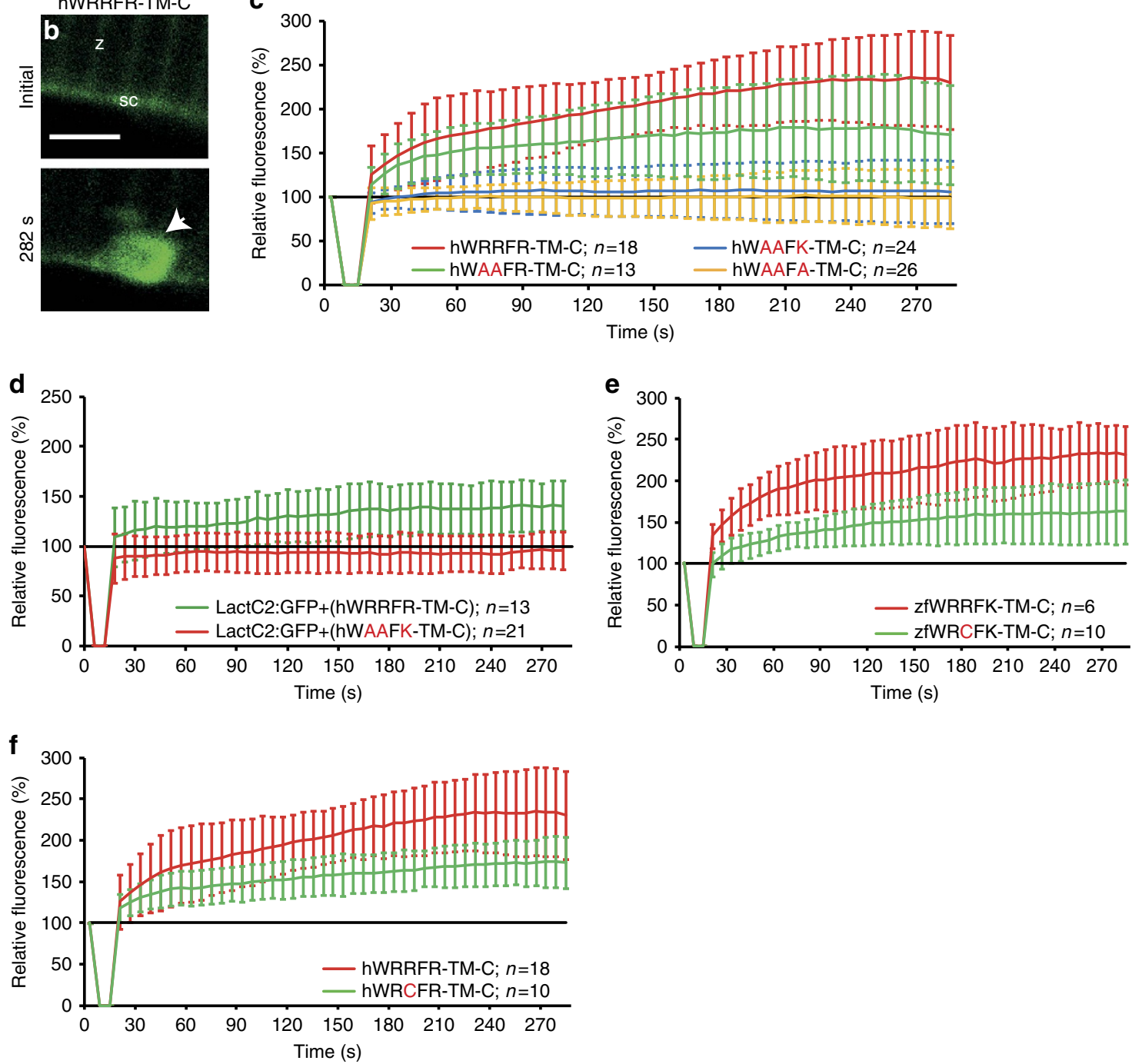

Figure 6 | Arginine-rich motif is mutated in myopathy patients. (a) Zebrafish zfWRRFK-TM-C and human hWRRFR-TM-C (red box arginine-rich motif; black box: TM domain, arrow: R2042C mutation). (b,c), hWRRFR-TM-C localized to the Z-line (z) and sarcolemma (sc) before damage (b). On damage, it accumulated at the lesion (b, arrow; c, red). In contrast, hWAAFK-TM-C (c, blue) and hWAAFA-TM-C (c, yellow) mutants did not accumulate and hWAAFR-TM-C caused significantly reduced accumulation (c, green). (d) LactC2:RFP accumulated in Dysf-KD myofibers when hWRRFR-TM-C (green) but not when mutant hWAAFK-TM-C (red). was co-expressed. (e,f) zfWRCFK-TM-C (e, green) and hWRCFR-TM-C (f, green), modelling the R2042C dysferlinopathic mutation showed significantly reduced accumulation (red). The fluorescence intensity of Dysf reporters at lesion relative to levels before damage is expressed as mean \pm s.d. $(n \geq 6)$. Note that the control data on hWRRFR-TM-C (c,f) are identical. Similarly, zfWRRFK-TM-C data (e) are also shown in Figs $3 \mathrm{~h}$ and $5 \mathrm{a}$. Scale bar, $4 \mu \mathrm{m}$.

reduced accumulation compared with hWRRFR-TM-C, yet, enrichment was not completely abolished, suggesting the third arginine may contribute to the activity (Fig. 6c, Supplementary Fig. 7a). On replacement of the third arginine by alanine (hWAAFA-TM-C) or lysine (hWAAFK-TM-C), accumulation was strongly impaired (Fig. 6c, Supplementary Fig. 7a).

Next, we knocked-down $d y s f$ and asked whether hWRRFR-TM$C$ rescued PS accumulation. Indeed, the PS sensor accumulated when hWRRFR-TM-C, but not when mutant hWAAFK-TM-C was co-expressed (Fig. 6d, Supplementary Fig. 7b).
Patients who presented with LGMD2B/MM were reported to have a point mutation in one of the arginines (R2042C, WRCFR $)^{33}$. Thus, we mutated the corresponding arginine to cysteine (zfWRCFK-TM-C, hWRCFR-TM-C). In agreement with our mutant analysis (Figs $5 \mathrm{a}$ and $6 \mathrm{c}$ ), this modification did not totally abolish, but significantly reduced accumulation of the zebrafish and the human DYSF reporter (Fig. 6e,f, Supplementary Fig. 7c-d). Hence, a likely cause of LGMD2B in these patients is inefficient DYSF and PS accumulation in the repair patch. 


\section{Discussion}

Lesions in the sarcolemma rapidly lead to formation of repair patches, preventing break-down of the sarcolemmal barrier and cell death. This repair patch has to be removed for restoration of the normal lipid bilayer structure of the sarcolemma. Several mechanisms have been previously suggested for patch removal, including membrane shedding, autophagy and endocytosis ${ }^{11,34,35}$. We show here that macrophages are key players in membrane repair by phagocytosing the repair patch. First, macrophages associate with and phagocytose material from the membrane patch. Second, knock-down of macrophages abolishes repair patch removal. Third, the repair patch rapidly and selectively accumulates PS a known 'eat-me' signal for macrophages ${ }^{36}$. PS is presented at the extracellular side of the repair patch, and thus is accessible to macrophages passing by. Moreover, phagocytosis is delayed when PS is masked.

Repair patch removal by macrophages can occur within minutes in some instances, whereas the clog persisted for hours in other cases. Thus, the repair patch does not seem to produce a long-range attractive signal pointing at the importance of local scanning by macrophages. Whereas small lesions were efficiently repaired, a characteristic sequence of events occurred for larger lesions: formation of a large repair patch, eventually sliding of the repair patch, secondary influx of $\mathrm{Ca}^{2+}$, contraction of the muscle cell, precipitation of AnxAs onto internal membranes, cell death and removal of dead cells by macrophages. Surprisingly, neutrophils were not involved in phagocytosis of repair patches or dead cells. We observed in all cases, however, recruitment of neutrophils to muscle injury by stabbing with a glass needle or tailfin cuts. This suggests that inflammatory signals may play additional roles. The overall much higher inflammatory status may explain the difference to myopathic human skeletal muscle, where neutrophils are heavily engaged ${ }^{37}$.

Among the lipids tested, only PS and cholesterol accumulated rapidly at lesions, whereas enrichment of general membrane markers was not observed. Our data underscore lipid sorting as mechanism for early repair patch assembly and suggest a key involvement of Dysf in PS-recruitment. Myofibers lacking Dysf failed to accumulate PS. Single-molecule tracking in Dysf morphant and control embryos showed Dysf-dependent, directed movement of PS to the site of lesion. Expression of Dysf fragments in HeLa cells and Dysf-depleted embryos causes PS enrichment. Hence, Dysf sorts PS to the repair patch. It remains to be uncovered whether cholesterol sorting employs the same mechanism.

We noted other lipids, even though not as fast as PS and cholesterol, to accumulate gradually over time. For example, $\mathrm{PI}(3,4) \mathrm{P}_{2}$ starts to build up in a delayed manner $4-5$ min post lesioning (Supplementary Fig. 7e). This likely reflects membrane flowing in from the edges and providing lipid material to eventually seal the sarcolemmal hole. In this context, systemic exocytosis may be involved expanding the membrane surface of a cell in response to membrane lesions ${ }^{2}$.

Binding of PS is mediated by the C2A-domains of Dysf (ref. 38). Our data suggest that the arginine-rich motif identified here plays an important additional role in PS transport. Arginine-rich peptides are known to bind PS (ref. 31). Thus, PS may thus directly interact with the Dysf arginine-rich motif.

Mutation of the arginine-rich motif affects localization of Dysf reporters. They appear to be located in the cytoplasm of uninjured myofibers. This suggests that Dysf has to be localized correctly to the sarcolemma to allow PS accumulation. Our single-molecule tracking data reveal that Dysf moves in the plane of the sarcolemma or immediately adjacent and parallel to it. A potential source of Dysf and PS might be caveolae which unfold on membrane stress and thus rapidly increase membrane surface $^{39}$. We did, however, not observe enrichment of general membrane markers at the lesion excluding overall flux of membrane material towards the lesion as mechanism of PS transport. The mechanism how Dysf and PS are transported towards the lesion remains elusive and needs further investigation. Another key question is how PS is incorporated in the repair patch so that it is exposed to the extracellular space. High local $\mathrm{Ca}^{2+}$ concentrations may inactivate flippases at the lesion as previously proposed in a different context ${ }^{40}$.

Zebrafish and human DYSF both contain arginine-rich motifs $\mathrm{N}$-terminally of the TM domain. hWRRFR-TM-C accumulated at the membrane patch and rescued PS accumulation in injured zebrafish myofibers, suggesting that these particular functions of Dysf have been conserved during evolution. This claim is supported by complementary approaches expressing the zebrafish Dysf reporter in HeLa cells; zfWRRFK-TM-C accumulated and mediated enrichment of PS at the site of lesion in human cells.

LGMD2B/MM is correlated with a mutation in the argininerich motif (R2042C) in human patients ${ }^{33}$. In accordance, modelling this mutation in both zebrafish and human DYSF fragments revealed significantly lower accumulation at membrane lesions. A disease causing effect of this mutation in humans is thus in complete agreement with our data. Taken together, these human genetic data underscore the physiological significance of the mechanism underlying membrane repair that we uncovered.

\section{Methods}

Zebrafish strains. The $\mathrm{AB}_{2} \mathrm{O}_{2}$ WT line (European Zebrafish Resource Centre EZRC, Karlsruhe) was used for all experiments. The transgenic lines $T g(m p e g 1: G F P)$ and $T g(L y s C: d s R E D)$ were a gift from the Lieschke and the Crosier labs, respectively ${ }^{14,15}$. Zebrafish husbandry ${ }^{41}$ and experimental procedures were performed in accordance with German animal protection regulations (Regierungspräsidium Karlsruhe, Germany, AZ35-9185.81/G-137/10).

Expression plasmids and sensors. Cloning was carried out following standard procedures (Supplementary Methods). Muscle expression of sensors was driven by the unc $45 b$ promoter (ref. 42). The lipid sensors LactC2:GFP, LactC2:RFP, GFP-2 $\times P H(P L C \delta), A K T-P H: E G F P$, Lyn-tailed mCherry-SEpHluorin ${ }^{20,23,24,26}$, secreted human Annexin V-YFP reporter ${ }^{28}$, human DYSF-Venus ${ }^{43}$, pcDNA3-Clover ${ }^{44}$ and $p E G F P:: 2 F Y V E-G F P^{25}$ were described previously. Please refer to Supplementary Table 2 for a summary of the sensors used.

Real-time imaging of membrane repair. Plasmids encoding sensors, BODIPY-cholesterol and TopFluor-PS (Avanti Polar Lipids, Alabaster, AL) were injected into the yolk of $1-2$ cell embryos ${ }^{45}$. Levels of expression of sensors did not affect the kinetics of membrane repair processes. Sarcolemmal lesions were generated using 3- to 5-day-old embryos, which were immobilized on a microscopy slide using $0.5 \%$ low melting point agarose supplemented with $0.02 \%$ MESAB. Embryos were imaged with a water dip-in $\times 63$ objective (NA: 0.90; HCX APO water; Leica) and installed at a Leica TCS SP2 confocal microscope and the corresponding Leica LCS software. The observations were performed at room temperature. The sarcolemma was damaged with a two-photon laser set to $860 \mathrm{~nm}$. Sensor accumulation at the membrane lesion was measured by determining the fluorescence intensity at the lesion in at least five independent experiments. Significance was tested with Welch's test followed by Bonferroni correction using MATLAB.

For imaging (16-22 h), multiple embryos were embedded in LMP agarose $(0.5 \%)$ in a $6 \mathrm{~cm}$ petridish and covered with $10 \mathrm{ml} 1 \times$ E3-medium (Supplementary Methods) containing $0.02 \%$ MESAB and $0.003 \%$ phenylthiourea (PTU). Individual damaged cells were imaged sequentially as Z-stacks of $40-90 \mu \mathrm{m}$ overnight under an upright TCS SP5 confocal microscope (Leica, HCX PL APO $\times 20 / 0.70$ lambda blue IMM CORR or HCX APO L $\times 40 / 0.80 \mathrm{~W}$ U-V-I objectives), using the brightfield and fluorescence channels $(488 \mathrm{~nm} / 561 \mathrm{~nm})$.

Knock-down and knock-out. Morpholinos against $d y s f \mathrm{mRNA}^{7}$ and for depleting macrophages ${ }^{16-18}$ were used as described $(0.8 \mathrm{mM} d y s f-M O, 0.5 \mathrm{mM}$ pu.1-MO; $0.5 \mathrm{mM}$ gcsfr-MO; $0.6 \mathrm{mM}$ irf8-MO). dysf and irf8 mutants were created by CRISPR/Cas9-mediated stop codon cassette insertion ${ }^{46}$.

Imaging of HeLa cells. HeLa cells were transfected using Lipofectamine 3000 (Thermo Fisher Scientific, Carlsbad, CA). After $24 \mathrm{~h}$ culture at $37^{\circ} \mathrm{C}$, cells were washed with phosphate buffered saline and imaged in DMEM containing $10 \%$ foetal bovine serum. Cells were imaged at $37^{\circ} \mathrm{C}$ on an Andor Revolution XD 
spinning disk confocal laser scanning microscope (BFi OPTiLAS, München, Germany) with an OLYMPUS ApoN60 × /1.49 oil immersion objective. The cell membrane was damaged by using the FRAPPA unit to irradiate a region of $6 \times 6$ pixels with $405 \mathrm{~nm}$ laser light at $200 \mu \mathrm{W}$ on the specimen with a pixel dwell time of $800 \mu$ s. Irradiation was repeated 600 times to locally damage the membrane within $18 \mathrm{~s}$. Image sequences were acquired in $10 \mathrm{~s}$ intervals, with the first image taken $20 \mathrm{~s}$ after membrane damage, followed by analysis with ImageJ.

Super-resolution localization microscopy. Zebrafish myofibers were imaged on a custom-built widefield inverted microscope (Axiovert 200, Zeiss, Göttingen, Germany) with single-molecule sensitivity ${ }^{47}$, equipped with a C-Apochromat, $\times 63 / 1.2$ W Corr objective (Zeiss), multiple excitation lasers (405, 473 and $561 \mathrm{~nm}$ ), an image splitter (Optosplit, Cairn Research Ltd, Faversham, UK) and an EMCCD camera (Ixon Ultra 897, Andor, Belfast, Northern Ireland). Embryos (4-5 dpf) anesthetized with $0.02 \%$ MESAB were immobilized on cover glass surfaces in 1\% LMP agarose; another cover slip on top held the embryos closely to the bottom surface. Muscle cell membranes were damaged by focusing $405-\mathrm{nm}$ laser light ( $5 \mathrm{~mW}$ on the specimen) for $2-4 \mathrm{~s}$. mEosFP was photoconverted to its red emitting form by $405 \mathrm{~nm}$ light and excited at $561 \mathrm{~nm}$. Image stacks were analysed using a-livePALM software ${ }^{48}$ (Supplementary Methods).

Data availability. All data are provided in the Supplementary Information.

\section{References}

1. Bansal, D. et al. Defective membrane repair in dysferlin-deficient muscular dystrophy. Nature 423, 168-172 (2003).

2. Cooper, S. T. \& McNeil, P. L. Membrane repair: mechanisms and pathophysiology. Physiol. Rev. 95, 1205-1240 (2015).

3. Bashir, R. et al. A gene related to Caenorhabditis elegans spermatogenesis factor fer-1 is mutated in limb-girdle muscular dystrophy type 2B. Nat. Genet. 20, 37-42 (1998).

4. Liu, J. et al. Dysferlin, a novel skeletal muscle gene, is mutated in Miyoshi myopathy and limb girdle muscular dystrophy. Nat. Genet. 20, 31-36 (1998).

5. Therrien, C., Di Fulvio, S., Pickles, S. \& Sinnreich, M. Characterization of lipid binding specificities of dysferlin $\mathrm{C} 2$ domains reveals novel interactions with phosphoinositides. Biochemistry 48, 2377-2384 (2009).

6. Lek, A. et al. Calpains, cleaved mini-dysferlinC72, and L-type channels underpin calcium-dependent muscle membrane repair. J. Neurosci. 33, 5085-5094 (2013).

7. Roostalu, U. \& Strahle, U. In vivo imaging of molecular interactions at damaged sarcolemma. Dev. Cell 22, 515-529 (2012).

8. Gerke, V., Creutz, C. E. \& Moss, S. E. Annexins: linking Ca2 + signalling to membrane dynamics. Nat. Rev. Mol. Cell Biol. 6, 449-461 (2005).

9. Lennon, N. J. et al. Dysferlin interacts with annexins A1 and A2 and mediates sarcolemmal wound-healing. J. Biol. Chem. 278, 50466-50473 (2003).

10. Bandorowicz-Pikula, J., Wos, M. \& Pikula, S. Do annexins participate in lipid messenger mediated intracellular signaling? A question revisited. Mol. Membr. Biol. 29, 229-242 (2012).

11. Idone, V. et al. Repair of injured plasma membrane by rapid $\mathrm{Ca} 2+$-dependent endocytosis. J. Cell Biol. 180, 905-914 (2008).

12. Reddy, A., Caler, E. V. \& Andrews, N. W. Plasma membrane repair is mediated by $\mathrm{Ca}(2+)$-regulated exocytosis of lysosomes. Cell 106, 157-169 (2001).

13. Akerboom, J. et al. Optimization of a GCaMP calcium indicator for neural activity imaging. J. Neurosci. 32, 13819-13840 (2012).

14. Ellett, F., Pase, L., Hayman, J. W., Andrianopoulos, A. \& Lieschke, G. J. mpeg1 promoter transgenes direct macrophage-lineage expression in zebrafish. Blood 117, e49-e56 (2011).

15. Hall, C. et al. The zebrafish lysozyme C promoter drives myeloid-specific expression in transgenic fish. BMC. Dev. Biol. 7, 42 (2007).

16. Rhodes, J. et al. Interplay of pu.1 and gatal determines myelo-erythroid progenitor cell fate in zebrafish. Dev. Cell 8, 97-108 (2005).

17. Liongue, C. et al. Zebrafish granulocyte colony-stimulating factor receptor signaling promotes myelopoiesis and myeloid cell migration. Blood 113, 2535-2546 (2009).

18. Li, L., Jin, H., Xu, J., Shi, Y. \& Wen, Z. Irf8 regulates macrophage versus neutrophil fate during zebrafish primitive myelopoiesis. Blood 117, 1359-1369 (2011).

19. Marguet, D., Luciani, M. F., Moynault, A., Williamson, P. \& Chimini, G. Engulfment of apoptotic cells involves the redistribution of membrane phosphatidylserine on phagocyte and prey. Nat. Cell Biol. 1, 454-456 (1999).

20. Yeung, T. et al. Membrane phosphatidylserine regulates surface charge and protein localization. Science 319, 210-213 (2008).

21. Leventis, P. A. \& Grinstein, S. The distribution and function of phosphatidylserine in cellular membranes. Annu. Rev. Biophys. 39, 407-427 (2010).

22. Hancock, J. F., Magee, A. I., Childs, J. E. \& Marshall, C. J. All ras proteins are polyisoprenylated but only some are palmitoylated. Cell 57, 1167-1177 (1989).
23. Koivusalo, M. et al. Amiloride inhibits macropinocytosis by lowering submembranous $\mathrm{pH}$ and preventing Racl and Cdc42 signaling. J. Cell Biol. 188, 547-563 (2010).

24. Fairn, G. D., Hermansson, M., Somerharju, P. \& Grinstein, S. Phosphatidylserine is polarized and required for proper Cdc42 localization and for development of cell polarity. Nat. Cell Biol. 13, 1424-1430 (2011).

25. Vieira, O. V. et al. Distinct roles of class I and class III phosphatidylinositol 3-kinases in phagosome formation and maturation. J. Cell Biol. 155, 19-25 (2001).

26. Kwon, Y., Hofmann, T. \& Montell, C. Integration of phosphoinositide- and calmodulin-mediated regulation of TRPC6. Mol. Cell. 25, 491-503 (2007).

27. Zhu, H. et al. Polymerase transcriptase release factor (PTRF) anchors MG53 protein to cell injury site for initiation of membrane repair. J. Biol. Chem. 286, 12820-12824 (2011).

28. van Ham, T. J., Mapes, J., Kokel, D. \& Peterson, R. T. Live imaging of apoptotic cells in zebrafish. Faseb. J. 24, 4336-4342 (2010).

29. Sapay, N., Guermeur, Y. \& Deleage, G. Prediction of amphipathic in-plane membrane anchors in monotopic proteins using a SVM classifier. BMC bioinformatics 7, 255 (2006).

30. Wiedenmann, J. \& Nienhaus, G. U. Live-cell imaging with EosFP and other photoactivatable marker proteins of the GFP family. Expert. Rev. Proteomics. 3, 361-374 (2006).

31. Nakai, Y., Nomura, Y., Sato, T., Shiratsuchi, A. \& Nakanishi, Y. Isolation of a Drosophila gene coding for a protein containing a novel phosphatidylserine-binding motif. J. Biochem. 137, 593-599 (2005).

32. Rosenbloom, K. R. et al. ENCODE data in the UCSC Genome Browser: year 5 update. Nucleic. Acids. Res. 41, D56-D63 (2013).

33. Blandin, G. et al. UMD-DYSF, a novel locus specific database for the compilation and interactive analysis of mutations in the dysferlin gene. Hum. Mutat. 33, E2317-E2331 (2012).

34. Jimenez, A. J. et al. ESCRT machinery is required for plasma membrane repair. Science 343, 1247136 (2014).

35. Salminen, A. \& Vihko, V. Autophagic response to strenuous exercise in mouse skeletal muscle fibers. Virchows. Arch. B Cell Pathol. Incl. Mol. Pathol. 45, 97-106 (1984).

36. Fadok, V. A. et al. Exposure of phosphatidylserine on the surface of apoptotic lymphocytes triggers specific recognition and removal by macrophages. J. Immunol. 148, 2207-2216 (1992).

37. Tidball, J. G. \& Villalta, S. A. Regulatory interactions between muscle and the immune system during muscle regeneration. Am. J. Physiol. Regul. Integr. Comp. Physiol. 298, R1173-R1187 (2010).

38. Davis, D. B., Doherty, K. R., Delmonte, A. J. \& McNally, E. M. Calcium-sensitive phospholipid binding properties of normal and mutant ferlin C2 domains. J. Biol. Chem. 277, 22883-22888 (2002).

39. Sinha, B. et al. Cells respond to mechanical stress by rapid disassembly of caveolae. Cell 144, 402-413 (2011).

40. Segawa, K. et al. Caspase-mediated cleavage of phospholipid flippase for apoptotic phosphatidylserine exposure. Science 344, 1164-1168 (2014).

41. Westerfield, M. The zebrafish: a guide for the laboratory use of zebrafish (Brachydanio reriro). (University of Oregon, 1993).

42. Etard, C. et al. Loss of function of myosin chaperones triggers Hsf1-mediated transcriptional response in skeletal muscle cells. Genome. Biol. 16, 267 (2015).

43. Covian-Nares, J. F., Koushik, S. V., Puhl, 3rd H. L. \& Vogel, S. S. Membrane wounding triggers ATP release and dysferlin-mediated intercellular calcium signaling. J. Cell. Sci. 123, 1884-1893 (2010).

44. Lam, A. J. et al. Improving FRET dynamic range with bright green and red fluorescent proteins. Nat. Methods 9, 1005-1012 (2012).

45. Holtta-Vuori, M. et al. BODIPY-cholesterol: a new tool to visualize sterol trafficking in living cells and organisms. Traffic 9, 1839-1849 (2008).

46. Gagnon, J. A. et al. Efficient mutagenesis by Cas9 protein-mediated oligonucleotide insertion and large-scale assessment of single-guide RNAs. PLOS ONE 9, e98186 (2014).

47. Hedde, P. N., Fuchs, J., Oswald, F., Wiedenmann, J. \& Nienhaus, G. U. Online image analysis software for photoactivation localization microscopy. Nat. Methods 6, 689-690 (2009).

48. Li, Y., Ishitsuka, Y., Hedde, P. N. \& Nienhaus, G. U. Fast and efficient molecule detection in localization-based super-resolution microscopy by parallel adaptive histogram equalization. ACS Nano 7, 5207-5214 (2013).

\section{Acknowledgements}

We thank N. Borel and the fish house team, M. Rastegar for microscopy and S. Grinstein, S. Vogel, M. Lin, L.C. Cantley, C. Etard, T. van Ham and R. Peterson for plasmids. We thank T. Dickmeis for critically reading the manuscript. US and GUN were funded by HGF, DFG (STR 439/8-1, Ni 291/12-1), EC IP ZF-HEALTH and BMBF-MIE. In addition, this research work is part of the project "Molecular Interaction Engineering: From Nature s Toolbox to Hybrid Technical Systems", which is funded by the German Federal Ministry of Education and Research (BMBF), funding code 031A095 C. 


\section{Author contributions}

V.M., L.Z., G.U.N and U.S designed the experiments V.M. and L.Z. performed experiments. V.M., U.S. and G.U.N wrote the manuscript. M.R. performed statistical analysis. C.G. provided transgenic zebrafish lines. M.S. helped to clone the calcium sensor. M.T. and T.B. contributed to experimental design and cloning. U.R. provided plasmids, S.R. reviewed the manuscript.

\section{Additional information}

Supplementary Information accompanies this paper at http://www.nature.com/ naturecommunications

Competing financial interests: The authors declare no conflict of interests.

Reprints and permission information is available online at http://npg.nature.com/ reprintsandpermissions/
How to cite this article: Middel, V. et al. Dysferlin-mediated phosphatidylserine sorting engages macrophages in sarcolemma repair. Nat. Commun. 7:12875 doi: $10.1038 /$ ncomms12875 (2016)

(c) (i) This work is licensed under a Creative Commons Attribution 4.0 International License. The images or other third party material in this article are included in the article's Creative Commons license, unless indicated otherwise in the credit line; if the material is not included under the Creative Commons license, users will need to obtain permission from the license holder to reproduce the material. To view a copy of this license, visit http://creativecommons.org/licenses/by/4.0/

(C) The Author(s) 2016 